\title{
Organising pneumonia - the first manifestation of rheumatoid arthritis
}

\author{
Desislava Kalinova, Zlatimir Kolarov, Rasho Rashkov \\ MHAT “St. Ivan Rilski”, Clinic of Rheumatology, Medical University, Sofia, Bulgaria
}

\begin{abstract}
Organising pneumonia (OP) is a distinct type of interstitial lung disease, because it can also be seen in association with several conditions such as infections, drugs, and connective tissue diseases. An association of OP with rheumatoid arthritis (RA) has also been described. Joint manifestations of RA usually precede lung involvements by several years; however, in less than $10 \%$ of cases of RA, interstitial lung disease may be the initial feature of RA. Organising pneumonia as the initial manifestation or developed simultaneously of RA is extremely rare, and its clinical features remain unknown. We present a 56-year-old woman with OP as the first manifestation of RA.
\end{abstract}

Key words: organizing pneumonia, rheumatoid arthritis, interstitial lung disease.

\section{Introduction}

Rheumatoid arthritis (RA) is a common inflammatory joint disease, which is characterised by persistent synovitis of multiple joints and is also a systemic condition that often affects extra-articular organs. Approximately $40 \%$ of RA patients suffer from some type of extra-articular manifestations, including interstitial lung disease (ILD), during the course of their disease [1, 2]. The most frequent histopathological patterns that occur in rheumatoid arthritis include usual interstitial pneumonia (UIP), nonspecific interstitial pneumonia (NSIP), organising pneumonia (OP), and diffuse alveolar damage $[3,4]$. Recent studies using high-resolution computed tomography $(\mathrm{HRCT})$ reported prevalence of interstitial lung diseases in patients with RA (RA-ILD) at a range of 27-67\%; however, a large proportion of these RA-ILD patients were asymptomatic $[5,6]$. Compared to other types of ILD, such as usual interstitial pneumonia and non-specific pneumonia, organising pneumonia associated with RA is less common and has a much better prognosis [7-9]. Organising pneumonia is a specific type of interstitial lung disease, histologically characterised by the presence of intraluminal buds of granulation tissue within alveolar ducts and alveoli [10].
The authors present a clinical case of 56 -year-old woman who developed OP as the first manifestation of rheumatoid arthritis.

\section{Clinical case}

In March 2017, the patient, a 56-year-old woman, presented to the general practitioner with left-side thoracic back pain, non-productive cough, weight loss, fever $\left(38^{\circ}-38.2^{\circ} \mathrm{C}\right)$, abundant night sweats, and astheno-adynamic syndrome. A chest X-ray was made, as infiltrate in the left lower lobe was visualised. The patient was consulted with a pulmonologist, because a diagnosis of pneumonia in the left lower lobe was established. Antibiotic therapy with Ceftriaxone 2 g/IV/daily and Ciprofloxacin $400 \mathrm{mg} / \mathrm{IV} /$ daily was applied for seven days, followed by Cefixime $400 \mathrm{mg}$ per os for seven days. The following therapy had an insignificant effect.

The patient was consulted with a pulmonologist for the second time. The control chest X-ray showed reduced but persistent pulmonary infiltrate in the left lower lobe and areas of pulmonary consolidations in the third and $10^{\text {th }}$ segments. Mantoux test for tuberculosis was performed and it was negative, as well as a following QuantiFERON-TB Gold In-Tube (QFT-GIT). Serological

\section{Address for correspondence:}

Desislava Kalinova, MHAT “St. Ivan Rilski”, Clinic of Rheumatology, Medical University, Urvich Street 13, 1612 Sofia, Bulgaria,

e-mail: desi.kalinova@abv.bg

Submitted: 19.09.2017; Accepted: 27.11.2017 


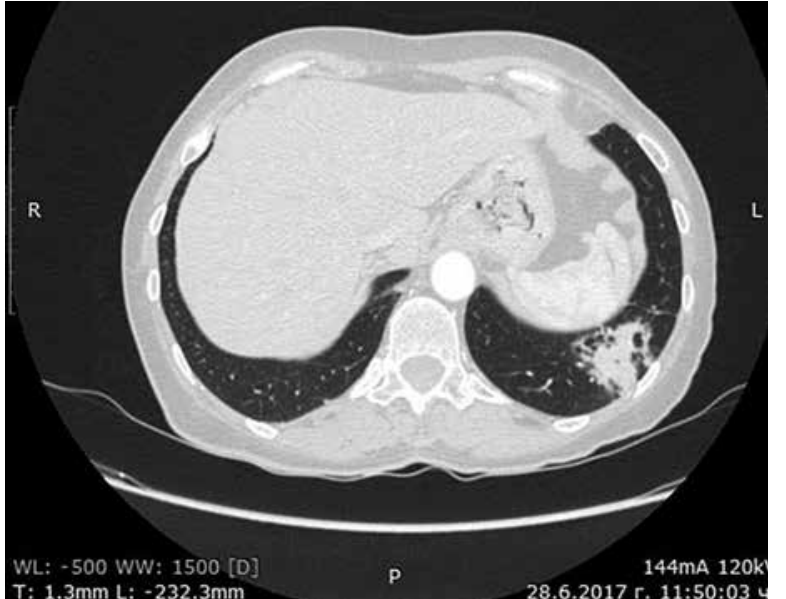

Fig. 1. Chest computed tomography. Two areas of sub-pleural and peribronchovascular consolidation in sixth and ninth segments, without fibrous changes.

tests for Mycoplasma pneumoniae (IgG, IgM, IgA) and Chlamydophila pneumonioae (IgG, IgA, IgM) were negative. Echocardiography did not show pathological changes. Therapy with Levofloxacin $500 \mathrm{mg} /$ per os for seven days was given without benefit. The patient had no history of medications, travel, or exposure to sick persons, pets, or other animals. The patient has never smoked. Sputum cultures yielded no pathogenic bacteria. Because of persistent clinical symptoms, chest computed tomography was made. Chest high-resolution computed tomography (HRCT) revealed pulmonary lesions, two areas with irregular form, and similar characteristic in the sixth and ninth segments, without fibrous changes, and without enlarged lymph nodes (Fig. 1). The conclusion of chest computed tomography was organising pneumonia (cryptogenic organising pneumonia).

The laboratory results were as follows: leukocytes 11.8 $\times 10^{9} /$; haemoglobin $126 \mathrm{~g} / \mathrm{l}$; erythrocytes $6.52 \times 10^{12} / \mathrm{l}$; platelets $558 \times 10^{9} /$; C-reactive protein (CRP) $63.4 \mathrm{mg} /$; erythrocyte sedimentation rate (ESR) $44 \mathrm{~mm}$; anti-cyclic
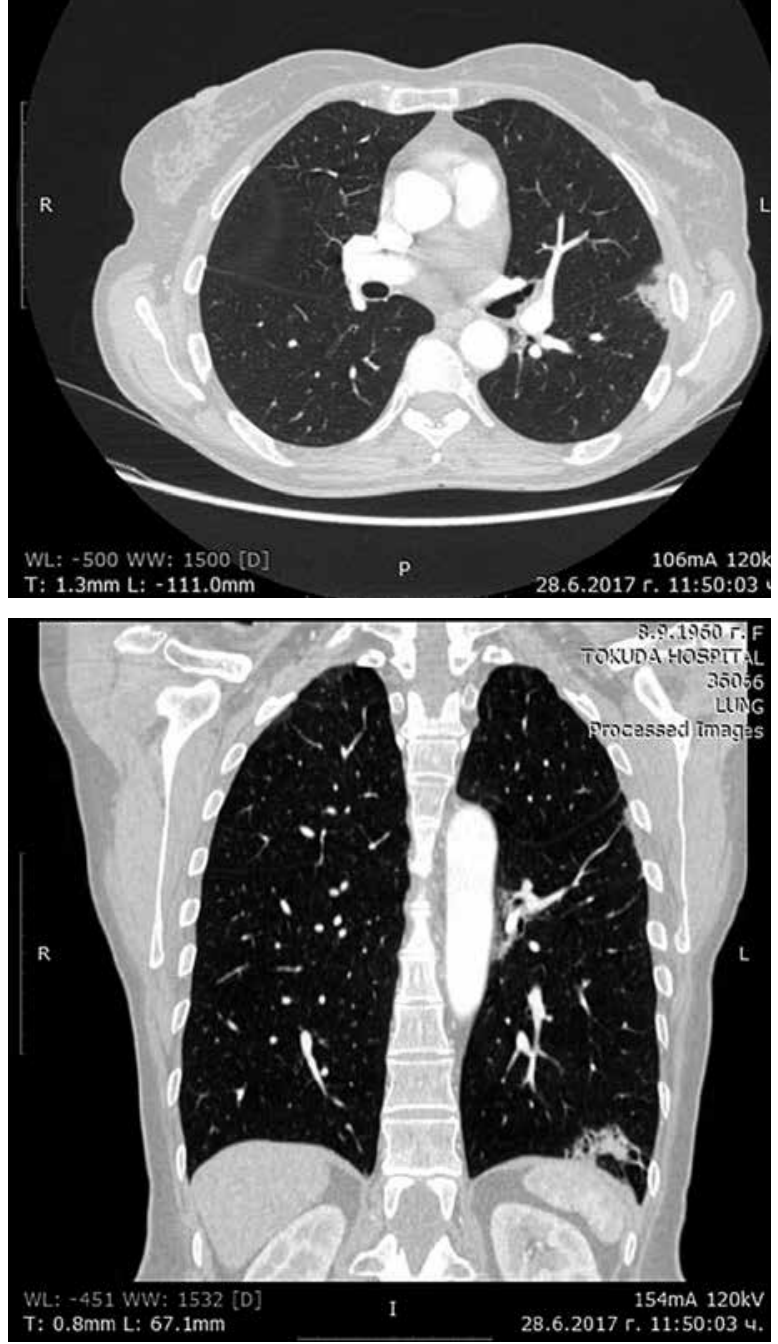

citrullinated peptide antibody (ACPA) > $250 \mathrm{U} / \mathrm{ml}$ (normal $<20 \mathrm{U} / \mathrm{ml}$ ); anti-mutated citrullinated vimentin antibody (anti-MCV) > $1000 \mathrm{U} / \mathrm{ml}$ (normal $<20 \mathrm{U} / \mathrm{ml}$ ); rheumatoid factor lgM $89.5 \mathrm{U} / \mathrm{ml}$ (normal $<20 \mathrm{U} / \mathrm{ml}$ ); rheumatoid factor lgG $63.6 \mathrm{U} / \mathrm{ml}$ (normal $<20 \mathrm{U} / \mathrm{ml}$ ); rheumatoid factor IgA $23.2 \mathrm{U} / \mathrm{ml}$ (normal < $20 \mathrm{U} / \mathrm{ml}$ ); ANA (IFI) 1 : 100 (normal < 1 : 100). Oral methylprednisolone $40 \mathrm{mg} /$ day was started, which resulted in rapid improvement of the symptoms. The corticosteroid dosage was tapered rapidly to $8 \mathrm{mg} /$ day.

Four months later, the patient developed arthritis, affecting small joints of the hands and the wrists. The patient was consulted with a rheumatologist. Ultrasound of the small joints of the hands and the contiguous tendons showed tenosynovitis of the flexor tendons with positive power Doppler signal and synovitis of the metacarpophalangeal joints. On X-ray of the hands, erosion of fourth metacarpophalangeal joint of right hand was noted (Fig. 2). A definite diagnosis of rheumatoid arthritis was made by a rheumatologist. 


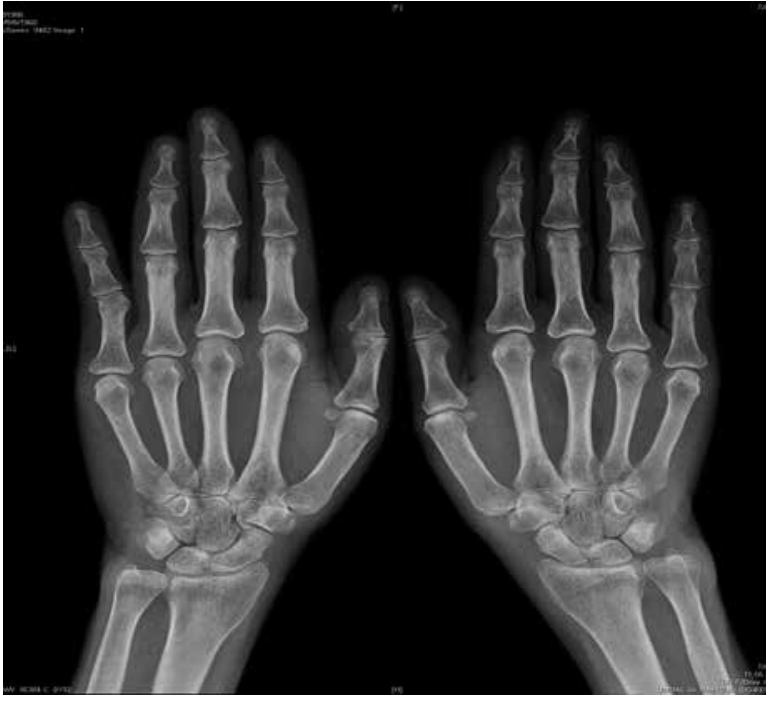

Fig. 2. Radiography of the hands. Erosion of the fourth metacarpophalangeal joint of the right hand was noted.

The methylprednisolone dosage was increased to $40 \mathrm{mg} /$ day and disease-modified antirheumatic drugs with methotrexate added. One month later the articular symptoms resolved and the ESR and CRP values normalised. The corticosteroid dosage was tapered slowly by $4 \mathrm{mg} /$ day every 10 days and methotrexate was continued.

\section{Discussion}

The authors present a clinical case of a patient who developed organising pneumonia as the first manifestation of rheumatoid arthritis. In RA, pulmonary involvement is one of the extra-articular manifestations. Four major patterns of computed tomography findings in RA patients were identified with usual interstitial pneumonia, non-specific interstitial pneumonia, bronchiolitis, and organising pneumonia [11]. The diagnosis of the organising pneumonia requires proving by histological examination. However, in specific clinical situations like the presence of clinical symptoms, the radiological picture and exclusion of other possible causes of often-migrated pulmonary infiltrates with an air bronchogram, the suggestion of OP is established [12]. Transbronchial lung biopsy is a very safe procedure with high probability of diagnosis, therefore it should be performed in all patients with OP suspicion. A similar radiological picture is observed in patients with MALT, pulmonary adenocarcinoma, or chronic eosinophilic pneumonia [12]. In the present case, a diagnosis of OP was based on the clinical and radiographic findings. The radiographic features of $\mathrm{OP}$ are characterised by multiple patchy airspace con- solidation, small nodular and linear opacities with subpleural and peribronchial distribution [13]. A lung biopsy with following pathological examination was not made because of refusal of the patient.

Only a few cases in which pulmonary manifestations preceded or simultaneously occurred with the onset of joint involvements have been described in the literature [14]. In the current clinical case the patient had no complex rheumatological assessment at the beginning of the disease, so it is impossible to clearly establish if the OP preceded or developed simultaneously with RA. In a case with OP, even without joint symptoms, serum anti-CCP should be tested because elevated anti-CCP autoantibodies are likely to manifest RA in the near future, reflecting its high disease activity [15]. The detection of serum anti-CCP Abs has proven to be a significant predictor of RA because of its higher specificity [16].

The prognosis of OP-associated RA is favourable because most patients rapidly respond to corticosteroid therapy [13]. Corticosteroid can mask joint symptoms necessary for early diagnosis, as with the present case. In RA, DMARD therapy should be initiated because it could provide more optimum therapeutic effect [17]. Thus, in a case with OP and positive ACPA, an early rheumatologic consultation should be taken into consideration to make an early decision to initiate DMARDs and glucocorticoids.

The authors declare no conflict of interest.

\section{References}

1. Cimmino MA, Salvarini C, Macchioni P, et al. Extra-articular manifestations in 587 Italian patients with rheumatoid arthritis. Rheumatol Int 2000; 19: 213-217.

2. Turesson C, O'Fallon WM, Crowson CS, et al. Extra-articular disease manifestations in rheumatoid arthritis: incidence trends and risk factors over 46 years. Ann Rheum Dis 2003; 62: 722-727.

3. Vij R, Noth I, Strek ME. Autoimmune-featured interstitial lung disease: a distinct entity. Chest 2011; 140: 1292-1299.

4. Tsuchiya J, Takayanagi N, Sigiura H, et al. Lung disease directly associated with rheumatoid arthritis and their relationship to outcome. Eur Respir J 2011; 37: 1411-1417.

5. Mori S, Cho I, Koga J, Sugimoto M. Comparison of pulmonary abnormalities on high-resolution computed tomography in patients with early versus longstanding rheumatoid arthritis. J Rheumatol 2008; 35: 1513-1521.

6. Doyle TJ, Dellaripa PF, Batra K, et al. Functional impact of a spectrum of interstial lung abnormalities in rheumatoid arthritis. Chest 2014; 146: 41-50.

7. Zou YQ, Li YS, Ding XN, Ying ZH. The clinical significance of HRCT in evaluation of patients with rheumatoid arthritis-associated interstitial lung disease: a report from China. Rheumatol Int 2012; 32: 669-673. 
8. Lee HK, Kim DS, Joo B, et al. Histopathological pattern and clinical features of rheumatoid arthritis-associated interstitial lung disease. Chest 2005; 127: 2019-2027.

9. Kim EJ, Collard HR, King TE. Rheumatoid arthritis-associated ILD: the relevance of histopathological and radiographic pattern. Chest 2009; 136: 1397-1405.

10. Colby TV. Pathological aspects of bronchiolitis obliterans organizing pneumonia. Chest 1992; 102; 38-43.

11. Mori S, Cho I, Koga J, et al. A simultaneous onset of organizing pneumonia and rheumatoid arthritis, along with a review of the literature. Mod Rheumatol 2008; 18: 60-66.

12. Baque-Juston $M$, Pellegrin A, Leroy $S$, et al. Organizing pneumonia: What is it? A conceptual approach and pictorial review. Diagn Interv Imaging 2014; 95: 771-777.

13. Izumi T, Kitaichi M, Nishimura K, et al. Bronchiolitis obliterans organizing pneumonia. Clinical features and differential diagnosis. Chest 1992; 102; 715-719.
14. Tanaka N, Kim JS, Newell JD, et al. Rheumatoid arthritis-related lung diseases: CT findings. Radiology 2004; 232: 81-91.

15. Hoshino C, Satoh N, Narita M, et al. Organizing pneumonia as the first manifestation of rheumatoid arthritis. BMJ Case Reports 2011; 2011: pii: bcr1120103558.

16. Lee DM, Schur Ph. Clinical utility of the anti-CCP assay in patients with rheumatic diseases. Ann Rheum Dis 2003; 62: 870-874.

17. Mori S. Management of rheumatoid arthritis patients with interstitial lung disease: Safety of Biological antirheumatic drugs and assessment of pulmonary fibrosis. Clin Med Insights Circ Respir Pulm Med 2015; 9: 41-49. 